\title{
Kombination mit Irinotecan - die Referenztherapie in der First-line-Therapie des fortgeschrittenen Kolorektalkarzinoms
}

\section{Combination with Irinotecan - the Reference First-Line Therapy for Advanced Colorectal Cancer}

\author{
H.W. Wilke \\ Klinik für Innere Medizin I und Internistische Onkologie/Hämatologie, Kliniken Essen Mitte, Essen
}

\section{Einleitung}

D urch randomisierte Studien ließen sich im vergangenen Jahrzehnt viele offene Fragen zur Chemotherapie des fortgeschrittenen Kolorektalkarzinoms beantworten. So wissen wir heute, dass die Chemotherapie sowohl in der First-line- als auch in der Second-line-Therapie zu signifikant besseren B ehandlungsergebnissen führt als eine rein supportive Therapie («best supportive care», BSC). Von Vorteil ist es, die Therapie frühzeitig zu beginnen anstatt zu warten, bis die zunehmende Symptomatik zu Therapiemaßnahmen zwingt. D es Weiteren haben die U ntersuchungen zur Therapie mit 5-Fluorouracil (5-FU) ergeben, dass die Kombination von 5-FU mit Folinsäure (FS) wirksamer ist als 5-FU alleine, dass 5-FU -Infusionsschemata wirksamer sind als 5-FU -B olusregime und 5-FU /F S-I nfusionsregime wirksamer als unmodulierte $5-\mathrm{FU}$-I nfusionsregime. Seit etwa 5 Jahren stehen uns neben 5-FU weitere Z ytostatika mit teilweise gutem W irkungspotenzial zur Verfügung. N eben dem neuen Platinderivat $\mathrm{Oxaliplatin,} \mathrm{den} \mathrm{oralen} \mathrm{5-FU-Prodrugs}$ U FT, Capecitabine und S1 sowie den spezifischen ThymidylatSynthasehemmern (z.B. Raltitrexed) hat insbesondere der Topoisomerase-I-H emmer I rinotecan der Therapie des fortgeschrittenen Kolorektalkarzinoms neue Impulse gegeben.

Phase-II-Studien ergaben für I rinotecan selbst in der Secondline-Situation nach Versagen einer vorausgegangenen 5-FU B ehandlung noch eine beachtliche A ktivität. E s war deshalb ein logischer Schritt, I rinotecan auch in der First-line-Therapie einzusetzen. In der Summe der vier Phase-II-M onotherapiestudien ergab sich dabei an insgesamt 175 Patienten eine objektive Remissionsrate von $26 \%$ (95\%-Konfidenzintervall 19-33\%). In den Vereinigten Staaten wurde dabei ein wöchentliches I rinotecan-Schema ( $125 \mathrm{mg} / \mathrm{m}^{2} /$ Woche), in den europäischen Studien ein 3wöchentliches Schema $\left(350 \mathrm{mg} / \mathrm{m}^{2}\right.$ ) eingesetzt. N ennenswerte $U$ nterschiede in der W irksamkeit der beiden Schemata waren nicht festzustellen. D ie mediane R emissionsdauer lag im Bereich von 8-9 M onaten, die mediane Ü berlebensdauer bei $12 \mathrm{M}$ onaten.
E s erschien nun aus mehreren $\mathrm{G}$ ründen vernünftig, I rinotecan und 5-FU in der First-line-Therapie zu kombinieren: Beide Substanzen besitzen eine signifikante klinische M onoaktivität, wobei in präklinischen Tumormodellen bei sequenzieller Verabreichung (Irinotecan vor 5-FU) eine synergistische Wirkungsverstärkung zu beobachten war. D ie beiden Wirkstoffe haben einen unterschiedlichen Wirkmechanismus, so dass auch eine $\mathrm{K}$ reuzresistenz weitgehend fehlt. A ußerdem haben 5-FU und I rinotecan mit A usnahme der D iarrhö wenig Ü berlappungen in ihren Toxizitätsspektren.

\section{Design der Studie V303}

In der multizentrischen randomisierten Phase-III-Studie V 303 wurde die Kombinationschemotherapie mit Irinotecan + 5-FU/FS mit dem bisherigen Standardregime 5-FU/FS verglichen [1]. A n dieser Studie beteiligten sich zahlreiche Zentren in E uropa, Israel und Südafrika. Primärer E ndpunkt der Studie war die Remissionsrate, sekundäre Endpunkte waren progressionsfreies Intervall, Z eit bis zum Therapieversagen, G esamtüberlebenszeit, Verträglichkeit der Therapie sowie L ebensqualität und andere Parameter des klinischen $B$ ehandlungserfolgs. Für den Kontrollarm und für die Kombination mit I rinotecan wurden zwei gängige hochdosierte 5-FU Infusionsregime zur Wahl gestellt, das 2wöchentliche Schema von de Gramont und das wöchentliche Schema der A rbeitsgemeinschaft Internistische 0 nkologie (AIO) der D eutschen $K$ rebsgesellschaft. Die teilnehmenden Z entren mussten sich vor Studienbeginn auf eines dieser Schemata festlegen.

387 Patienten mit messbarer Tumorerkrankung und einem WHO Performance-Status $\leq 2$, die im fortgeschrittenen Stadium noch keine Chemotherapie erhalten hatten (eine adjuvante Therapie musste mindesten $6 \mathrm{M}$ onate vorher beendet worden sein), wurden für diese Studie rekrutiert und zwischen den beiden Therapiearmen randomisiert (Tab. 1).

\begin{tabular}{ll}
\hline KARGER & ○ 2000 S. Karger G mbH , Freiburg \\
$\begin{array}{l}\text { Fax +49 761 4520714 } \\
\begin{array}{l}\text { E-mail Information@K arger.de } \\
\text { www.karger.com }\end{array}\end{array}$ & $\begin{array}{l}\text { A ccessible online at: } \\
\text { www.karger.com/journals/onk }\end{array}$
\end{tabular}

Prof. Dr. Hansjochen Wilke

$\mathrm{K}$ linik für Innere M edizin I und Internistische 0 nkologie/H ämatologie

K liniken E ssen-M itte

$\mathrm{H}$ enricistraße 92

D -45136 E ssen (G ermany) 
Wie den Patientenzahlen zu entnehmen ist (aufgeführt sind die tatsächlich behandelten Patienten), erfolgte bei etwa $3 \mathrm{~V}$ iertel der Patienten in beiden Studienarmen die 5-FU -Therapie nach dem Schema von de Gramont. Die Patientencharakteristika waren in beiden A rmen nahezu ausgeglichen. E twa 25\% der Patienten hatten eine adjuvante Vorbehandlung. Median 1 O rgan war metastatisch befallen (bei nahezu $80 \%$ der Patienten die L eber, bei etwa $25 \%$ die L unge).

Innerhalb des Kombinationsarms war die mediane Behandlungsdauer mit dem A IO - und de-G ramont-Schema nahezu gleich (24 bzw. 25 Wochen), im R eferenzarm etwas kürzer (21 Wochen mit A I O, 18 Wochen mit de G ramont). Dies entsprach jeweils median 3-4 Therapiezyklen. D ie relative D osisintensität war im Kombinationsarm mit dem A IO-Schema etwas geringer ( $82 \%$ für I rinotecan, $81 \%$ für $5-\mathrm{FU}$ ) als mit dem de-G ramont-Schema (93 bzw. 92\%), dieser U nterschied war jedoch nicht signifikant und wahrscheinlich auch klinisch nicht relevant.

\section{Verträglichkeit}

D ie hämatologische Toxizität war im Kombinationsarm höher als im Referenzarm (Neutropenie WHO Grad 3/4 bei 42 vs. $11 \%$ der Patienten bzw. in 20 vs. 5\% der Zyklen), doch trat eine febrile Neutropenie mit I rinotecan/5-FU/FS nur bei $5 \%$ der Patienten (1\% der Zyklen) und eine neutropenische Infektion nur bei $2 \%$ der Patienten ( $1 \%$ der Zyklen) auf. A uch eine Diarrhö Grad 3/4 war erwartungsgemäß mit der Kombinationstherapie häufiger (22\% der Patienten bzw. 7\% der Zyklen) als mit 5-FU/FS alleine (10 zu 4\%). A lle anderen Nebenwirkungen waren vergleichsweise selten. $D$ ie A lopezie erreichte höchstens $\mathrm{G}$ rad 2 ( 21 vs. 4\% der Patienten im Kombinationsvs. R eferenzarm).

\section{Wirksamkeit}

In Tabelle 2 sind die E rgebnisse zur Wirksamkeit beider Therapien gegenübergestellt. $\mathrm{D}$ ie $\mathrm{A}$ nsprechrate betrug mit der I rinotecan-Kombination 49\%, mit 5-FU/FS alleine nur 31\%
( $p<0,001)$. A lle R emissionen wurden nach 6-7 Wochen durch ein externes R eview-K omitee überprüft, wobei sich der signifikante U nterschied bestätigte ( 41 vs. $23 \%, p=0,005$ ). A uch die $D$ auer der Tumorwachstumskontrolle (objektive R emissionen und Tumorstillstand) war mit der Kombinationstherapie signifikant länger ( 8,6 vs. 6,2 M onate, $p<0,001$ ). Weiterhin ergaben sich signifikante Vorteile zugunsten der I rinotecan-Kombination beim progressionsfreien Intervall (6,7 vs. 4,4 Monate, $p<0,001)$, aber auch bei der medianen Ü berlebenszeit (17,4 vs. $14,1 \mathrm{M}$ onate, $p=0,031)$. D amit ist diese Studie die erste, die in der First-line-Therapie einen signifikanten Ü berlebensunterschied zwischen zwei geprüften Chemotherapie-R egimes nachweisen konnte. Die Ü berlebenswahrscheinlichkeit nach 1 Jahr betrug im Kombinationsarm 69,1\%, im Referenzarm nur $59,1 \%$.

U m auszuschließen, dass die leichten U ngleichverteilungen in den Patientencharakteristika einen E influss auf die B ehandlungsergebnisse hatten, wurde eine multivariate A nalyse durchgeführt. Sie ergab, dass die A rt der B ehandlung von allen untersuchten Variablen den signifikantesten E influss auf die R emissionsrate und das progressionsfreie Intervall hatte. D er 0 dds-R atio für die A nsprechrate betrug 2,564 zugunsten von Irinotecan/5-FU/FS, während das Progressionsrisiko unter 5-FU /FS um das 1,620fache höher lag als mit der Kombination. $E$ benfalls von B edeutung für das A nsprechen des Tumors war ein vorausgegangener $\mathrm{G}$ ewichtsverlust ( $>5 \%$ vs. $\leq 5 \%$ ) und die Zeit von der D iagnosestellung bis zum ersten A uftreten von $M$ etastasen, während das progressionsfreie I ntervall noch vom A Iter ( $\geq 58$ vs. $<58$ Jahre) und von einer L ebermetastasierung beeinflusst wurde. Weitere $C$ hemotherapien, die die Patienten nach einsetzender Tumorprogression erhielten, hatten keinen nennenswerten E influss auf die G esamtprognose.

\section{Lebensqualität}

D ie A uswertung der Verlaufskurven der L ebensqualität ergab keine signifikanten Unterschiede zwischen den beiden Studienarmen. In beiden A rmen war im Laufe der ersten 5-6 Monate der Studie eine leichte Besserung des Durchschnitts-Scores der L ebensqualität zu verzeichnen. D araus ist
Tab. 1 Studienarme der multizentrischen randomisierten Phase-III-Studie V 303 (mod. nach D ouillard et al. [1])

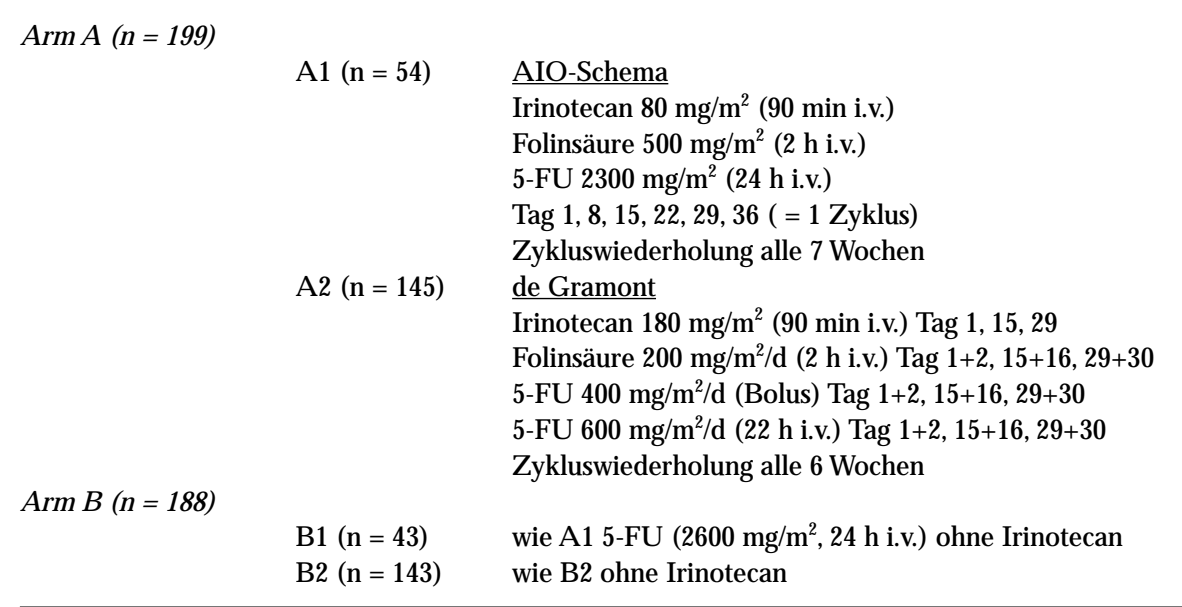


Tab. 2. E rgebnisse der Phase-III-Studie V 303 in der First-line-Therapie des fortgeschrittenen Kolorektalkarzinoms (mod. nach D ouillard et al. [1])

\begin{tabular}{lllr}
\hline & $\begin{array}{l}\text { Irinotecan }+ \\
\text { 5-FU/Folinsäure } \\
(n=169)\end{array}$ & $\begin{array}{l}\text { 5-FU/Folinsäure } \\
(n=169)\end{array}$ & $p$ \\
\hline A nsprechrate, \% & 49 & 31 & $<0,001$ \\
B estätigte A nsprechrate, \% & 41 & 23 & 0,005 \\
Vollständige R emission, \% & 4 & 0 & $<0,001$ \\
M ediane D auer CR/PR/SD, M onate & 8,6 & 6,2 & $<0,001$ \\
M edianes progressionsfreies Intervall, M onate & 6,7 & 4,4 & 0,031 \\
M edianes Ü berleben, M onate & 17,4 & 14,1 & \\
1-Jahres-Ü berlebenswahrscheinlichkeit, \% & 69,1 & 59,1 & \\
\hline
\end{tabular}

$\mathrm{CR}=$ vollständige, $\mathrm{PR}=$ partielle $\mathrm{R}$ emission, $\mathrm{SD}=$ stable disease. zu schließen, dass sich die häufigeren Nebenwirkungen mit dem Kombinationsregime nicht negativ auf die L ebensqualität auswirkten. E ine definitive Verschlechterung des L ebensqualitäts-Scores um $20 \%$ setzte in der I rinotecan/5-FU/F S-G ruppe sogar signifikant später ein als mit 5-FU/FS alleine $(p=0,04)$. In gleicher Weise wurde die Verschlechterung des Performance-Status signifikant verzögert $(p=0,046)$.

\section{Fazit}

D ie Studie V 303 zeigte eine klare Ü berlegenheit der Kombination I rinotecan/5-FU/FS gegenüber der bisherigen Standardtherapie in der First-line-Therapie des fortgeschrittenen Kolorektalkarzinoms, 5-FU/FS. D ie I rinotecan-Kombination induzierte signifikant mehr objektive R emissionen und verlängerte signifikant das progressionsfreie Intervall und die G esamtüberlebenszeit der Patienten. A ußerdem zögerte die $B$ ehandlung mit dem Kombinationsregime eine Verschlechterung der L ebensqualität und des A Ilgemeinzustandes deutlich hinaus. Die Nebenwirkungen der Irinotecan-Kombination waren beherrschbar, nicht kumulierend und reversibel und wirkten sich nicht negativ auf das A Ilgemeinbefinden der Patienten aus. In einer zweiten großen randomisierten amerika- nischen Studie, die ebenfalls die Kombination I rinotecan/ 5-FU/FS mit 5-FU/FS alleine verglich, konnte die Ü berlegenheit der Kombinations-Chemotherapie bestätigt werden [2]. A uch in dieser Studie war I rinotecan/5-FU/FS, bezogen auf die Induktion objektiver Remissionen, das progressionsfreie Ü berleben und die Ü berlebenszeit der alleinigen 5-FU/FSTherapie statistisch signifikant überlegen. D as neue Kombinationsregime von Irinotecan mit 5-FU/FS markiert daher einen substanziellen Fortschritt in der B ehandlung des Kolorektalkarzinoms und sollte zukünftig als Therapie der Wahl gelten und als R eferenztherapie für zukünftige Vergleichsstudien eingesetzt werden.

\section{Literatur}

1 D ouillard $J Y$, C unningham $D, R$ oth $A D, N$ avarro $M$, James $R D, K$ arasek $P$, J andik $P$, I veson $T$, Carmichael J, A lakl M , G ruia G, A wad L, R ougier P: I rinotecan combined with fluorouracil compared with fluorouracil alone as first-line treatment for metastatic colorectal cancer: A multicentre randomised trial. Lancet 2000;355: 1041-1047.

2 Saltz L B, D ouillard J, Pirotta N, A wad L, E Ifring G L, G ruia G, L ocker PM , A lakl M, $K$ night $D, M$ iller LL: Combined analysis of two phase III randomized trials comparing irinotecan $(C)$, fluorouracil $(F)$, leucovorin $(L)$ vs. $F$ alone as first-line therapy of previously untreated metastatic colorectal cancer (M CRC). Proc A m Soc Clin Oncol 2000;19:242a. 